\title{
Fatores associados a abandono precoce do tratamento em psicoterapia de orientação analítica
}

\author{
Factors related to early dropout in psychoanalytic psychotherapy
}

\author{
Simone Hauck ${ }^{1}$, Letícia Kruel ${ }^{2}$, Anne Sordi ${ }^{3}$, Gabriela Sbardellotto ${ }^{4}$, Aline Cervieri ${ }^{5}$, Laura \\ Moschetti $^{6}$, Sidnei Schestatsky ${ }^{7}$, Lucia Helena Freitas Ceitlin ${ }^{8}$
}

\begin{abstract}
${ }^{1}$ Médica psiquiatra. Especialista em Psicoterapia. Mestre em Psiquiatria, Universidade Federal do Rio Grande do Sul (UFRGS), Porto Alegre, RS. Doutoranda, Curso de Pós-Graduação em Ciências Médicas: Psiquiatria, UFRGS. ${ }^{2}$ Acadêmica de Psicologia, Pontifícia Universidade Católica do Rio Grande do Sul (PUCRS), Porto Alegre, RS. ${ }^{3}$ Acadêmica de Medicina, UFRGS. ${ }^{4}$ Acadêmica de Psicologia, PUCRS. ${ }^{5}$ Médica. ${ }^{6}$ Acadêmica de Medicina, UfRGS. Auxiliar de pesquisa, Programa de Psicoterapia Psicanalítica, HCPA. ${ }^{7}$ Médico psiquiatra. Mestre em Saúde Pública. Doutor em Psiquiatria. Professor adjunto, Departamento de Psiquiatria e Medicina Legal, Faculdade de Medicina, UFRGS. ${ }^{8}$ Médica psiquiatra. Mestre em Saúde Pública. Doutora em Psiquiatria. Professora adjunta, Departamento de Psiquiatria e Medicina Legal, Faculdade de Medicina, UFRGS.

Este estudo foi realizado no Serviço de Psiquiatria, Hospital de Clínicas de Porto Alegre (HCPA), Departamento de Psiquiatria e Medicina Legal, Universidade Federal do Rio Grande do Sul (UFRGS), Porto Alegre, RS, como parte do Programa de Pós-Graduação em Ciências Médicas da UFRGS: Psiquiatria.

Apoio financeiro: Fundo de Incentivo a Pesquisa e Ensino do Hospital de Clínicas de Porto Alegre (FIPE-HCPA).
\end{abstract}

\section{Resumo}

Introdução: A eficácia da psicoterapia psicanalítica foi bem estabelecida através de ensaios clínicos controlados; no entanto, algumas das características individuais que predizem melhores resultados são ainda pouco estudadas. O objetivo do estudo foi avaliar a associação entre os dados demográficos, o diagnóstico psiquiátrico, a sintomatologia clínica, a qualidade de vida, os critérios de indicação da psicoterapia, o estilo defensivo e o abandono da psicoterapia psicanalítica antes de 3 meses de tratamento.

Método: Uma amostra consecutiva de 56 pacientes foi avaliada após a indicação da psicoterapia, através de um protocolo padronizado, do World Health Organization Quality of Life Bref (WHOQOL-Bref), do Self Report Questionnaire, do Defensive Style Questionnaire, da Escala de Funcionamento Defensivo do Manual de Diagnóstico e Estatística das Perturbações Mentais, $4^{\mathrm{a}}$ edição, texto revisado (DSM-IV-TR) e da Avaliação do Funcionamento Global (Global Assessment of Functioning), e acompanhada por 3 meses.

Resultados: A taxa de abandono foi de 12,5\%. Não houve diferença quanto à Avaliação do Funcionamento Global, Self Report Questionnaire e Defensive Style Questionnaire. Os pacientes que abandonaram estavam satisfeitos com sua saúde, apesar da gravidade da sua psicopatologia, mesmo controlando para outras variáveis $(\mathrm{p}<0,0001)$. O grupo que permaneceu em tratamento apresentava melhor adaptação prévia e maior proporção de indivíduos com inteligência estimada como média ou superior (p < 0,05). Maior proporção de pacientes no grupo do abandono foi classificada em níveis imaturos, segundo a Escala de Funcionamento Defensivo do DSM-IV-TR.

Conclusões: A gravidade da psicopatologia, isoladamente, não teve associação com o abandono; entretanto, menor nível de insight e defesas mais imaturas (especialmente narcisistas) foram associados a maiores taxas de abandono. Esses aspectos devem ser seriamente considerados, juntamente com as expectativas do paciente quanto ao método analítico, e avaliados criteriosamente antes da indicação.

Descritores: Psicoterapia, psicanálise, desistência do paciente, resultado de tratamento, mecanismos de defesa, qualidade de vida. 


\section{Abstract}

Introduction: The efficacy of psychoanalytic psychotherapy is well established in controlled clinical trials; however, some individual characteristics that predict better outcomes are yet poorly studied. This study aimed at evaluating the association of demographics data, psychiatric diagnosis, clinical impairment, quality of life, aspects of psychotherapy suitability, defensive style and dropout before 3 months.

Method: A consecutive sample of 56 subjects was evaluated after psychotherapy indication through a standardized protocol, World Health Organization Quality of Life Bref (WHOQOL-Bref), Self Report Questionnaire, Defensive Style Questionnaire, Scale of Defensive Functioning of Diagnostic and Statistical Manual of Mental Disorders, fourth edition (DSM-IV-TR) and Global Assessment of Functioning, and followed for 3 months.

Results: Dropout rate was $12.5 \%$. There was no difference between groups in respect to Global Assessment of Functioning, Self Report Questionnaire and Defensive Style Questionnaire scores. Dropout patients reported to be satisfied with their health, despite psychopathological severity, even when other variables were controlled ( $<<0.0001)$. The group that remained in psychotherapy was better adjusted before treatment and had average or superior estimated intelligence $(\mathrm{p}<0.05)$. More dropout patients presented lower levels of defensive style, by means of the Scale of Defensive Functioning of DSM-IV-TR.

Conclusions: Psychopathology severity alone did not predict dropout. However, patients with lower levels of insight and immature defenses (especially narcissistic) had higher dropout rates. Therefore, such aspects must be seriously considered, along with patients' expectations about the psychoanalytic method, and should be judiciously assessed before indication.

Keywords: Psychotherapy, psychoanalysis, patient dropout, treatment outcome, defense mechanisms, quality of life.

\section{Introdução}

A partir do advento da psicanálise, diferentes modalidades terapêuticas foram desenvolvidas para tratar os transtornos mentais e problemas de natureza emocional. Dentre as opções terapêuticas, estão as diversas formas de psicoterapia e a psicofarmacologia. A eficácia dos diferentes métodos vem sendo sistematicamente testada, de forma que se possa adequar o tratamento às necessidades individuais dos pacientes e do sistema de saúde. Estudos que compararam a terapia cognitivo-comportamental com a psicoterapia psicodinâmica breve ou interpessoal de orientação psicodinâmica encontraram resultados equiparáveis em termos de eficácia geral. Entretanto, variações nos resultados têm sido apontadas quando levamos em consideração as características individuais dos pacientes, como tempo de doença, comorbidades, traços de personalidade, severidade, diagnóstico psiquiátrico, existência de foco, qualidade das relações de objeto, funcionamento defensivo, estado civil, entre outros. Esses resultados, porém, ainda são incipientes, e não há um consenso que permita definir qual o tipo de tratamento mais adequado para um paciente em particular, a partir de evidências científicas ${ }^{1-9}$.

Diversos autores vêm confrontando o mito de que todos os pacientes podem ser tratados e curados com psicoterapia de orientação analítica, ou análise, como um equívoco que pode desacreditar a técnica e levar a gastos e sofrimentos desnecessários ${ }^{10}$. O método analítico propõe uma ferramenta terapêutica que busca a melhora dos sintomas através da compreensão do funcionamento atual e dos aspectos inconscientes envolvidos no conflito. Esse processo não é isento de um "custo emocional" e pode ocasionar piora inicial dos sintomas, ao evidenciar características do paciente que contribuem para o estabelecimento e manutenção dos seus aspectos problemáticos. Nesse sentido, é preciso que haja alta motivação para se melhorar através do método analítico, que, muitas vezes, implica em objetivos mais amplos, como ampliar a capacidade da mente e as possibilidades de escolha do indivíduo. $\mathrm{O}$ paciente possuir capacidades de simbolização e abstração é certamente essencial para se indicar uma psicoterapia de orientação analítica.

Conseqüentemente, a indicação de um tratamento de orientação analítica, por definição, exige consideração de critérios psicológicos que não costumam ser avaliados em modelos tradicionais de pesquisa clínica. Paralelamente, tem sido questionada a adequação do modelo de eficácia, representado por estudos tipo ensaio clínico randomizado, para o delineamento de pesquisas nessa área. A aplicação de rigorosos critérios de seleção com o objetivo de homogeneizar a amostra e o controle estrito e sistemático da metodologia terapêutica acabam por inviabilizar a generalização dos resultados, por afastar substancialmente o tratamento testado daquele aplicado na "vida real" e desvirtuar aspectos da técnica que são essenciais à caracterização de um tratamento como de orientação analítica ${ }^{11}$. Estudos de efetividade que utilizem alguns parâmetros dos modelos de eficácia (com a intenção de preservar a validade interna), juntamente com um desenho naturalístico, seriam mais adequados ${ }^{10-15}$.

Muitas interrupções de tratamento, bem como desfechos pouco efetivos, podem decorrer, de fato, de avaliações iniciais inadequadas e indicações imprecisas. Freud (1913) afirma que “(...) no que concerne ao psicanalista, se o caso for desfavorável, o analista cometeu um erro prático: foi responsável por despesas desnecessárias e desacreditou seu método de tratamento"10,16. Ao estudar o fenômeno do abandono em psicoterapia, Urtiaga et al. encontraram que o risco 
de o paciente abandonar o tratamento nas quatro primeiras sessões é muito alto e que após a décima sessão o risco cai significativamente ${ }^{17}$. Nas instituições de ensino, fatores como a necessidade de trocas periódicas de terapeuta parecem explicar uma parte significativa dos abandonos ${ }^{18}$.

Algumas psicopatologias, como o transtorno de personalidade borderline, têm sido particularmente associadas à alta chance de interrupção: dois em cada cinco pacientes abandonam o tratamento, independentemente de quem é o terapeuta ${ }^{19}$. A presença de traços narcisistas importantes também implica em uma maior chance de interrupção, por inibir o estabelecimento de uma relação que fortaleça a aliança de trabalho ${ }^{20}$. Um estudo que acompanhou 43 pacientes em tratamento psicanalítico ambulatorial encontrou que maiores níveis de insight pré-tratamento estiveram associados à maior duração da psicoterapia, menores chances de abandono e busca por tratamento psicoterápico adicional em um seguimento de 4 anos $^{21}$.

Outros fatores sistematicamente associados ao resultado da psicoterapia são a motivação do paciente para o tratamento, capacidade de pensar psicologicamente, curiosidade psíquica, nível de sofrimento inicial e nível de organização da personalidade (força de ego, qualidade das relações objetais) ${ }^{9,22}$. A qualidade inicial da aliança terapêutica (ou aliança de trabalho) tem sido especialmente considerada, independentemente da técnica utilizada ${ }^{23-25}$.

A escassez de evidências quanto às características do paciente, associada a uma maior efetividade, colabora para a ausência de um consenso geral quanto aos critérios de indicação, aspecto de particular relevância quando falamos em termos de saúde pública. São necessários estudos que investiguem a eficácia dos programas de psicoterapia atualmente utilizados, no sentido de colaborar para o refinamento dos critérios e das decisões sobre alocação de recursos ${ }^{26}$. O objetivo deste trabalho foi investigar, em uma amostra de pacientes atendidos no Programa de Psicoterapia Psicanalítica do Serviço de Psiquiatria do Hospital de Clínicas de Porto Alegre (HCPA), a existência de associação entre abandono do tratamento nos primeiros 3 meses e características demográficas, gravidade clínica, funcionamento defensivo e critérios tradicionalmente associados à indicação da psicoterapia de orientação analítica.

\section{Metodologia}

\section{Amostra}

Uma amostra consecutiva de 56 pacientes que iniciaram o atendimento no Programa de Psicoterapia Psicanalítica do HCPA, em 2005, foi acompanhada por um período de 3 meses, a partir da indicação da psicoterapia de orientação analítica. Abandono precoce foi definido como a interrupção do tratamento (comunicada ou não) antes dos 3 meses. A escolha do tempo de seguimento foi baseada em evidências que apontam o período inicial da psicoterapia como associado a maiores taxas de abandono, que caem drasticamente a partir da $10^{\mathrm{a}}$ sessão ${ }^{17}$. Além disso, pode-se considerar que 12 semanas (ou 12 sessões nos tratamentos semanais) seria um tempo mínimo para que modificações obtidas em um tratamento de orientação analítica se reflitam na sintomatologia e na melhora do funcionamento. Desse modo, se diminuiria a chance de que o abandono tenha sido uma "alta dada pelo paciente", por atingir os seus próprios objetivos, independentes daqueles estabelecidos pelo terapeuta. Embora sua importância no estabelecimento do vínculo inicial e na correta indicação do método sejam reconhecidamente fundamentais, as entrevistas de avaliação não foram consideradas como tempo de tratamento, por não serem tecnicamente equivalentes às sessões de psicoterapia psicanalítica propriamente ditas.

\section{Indicação e características da psicoterapia}

A avaliação da indicação da psicoterapia foi guiada por um protocolo de avaliação especificamente desenvolvido para esse fim, a partir da revisão da literatura, reuniões com experts e estudos-piloto. Foram selecionados 21 critérios de indicação, englobando dados como disponibilidade de tempo, recursos do ego, existência de foco e motivação para a realização de um tratamento de orientação analítica (Tabela 1). Os critérios foram avaliados através de respostas dicotômicas tipo sim/não, a partir dos dados obtidos nas entrevistas de avaliação ${ }^{27}$. O conjunto dos critérios avaliados foi utilizado para determinar as indicações: nenhum fator independente acarretava a contra-indicação da psicoterapia. Além disso, foram avaliados dados sociodemográficos, diagnóstico segundo o Manual de Diagnóstico e Estatística das Perturbações Mentais, $4^{\mathrm{a}}$ edição, texto revisado ${ }^{28}$ (DSM-IV-TR), foco da psicoterapia, sentimentos despertados (transferenciais e contratransferenciais), defesas predominantes, hipótese psicodinâmica preliminar (conflito atual, funcionamento e conflito primário), uso de psicofármacos, características da psicoterapia (supervisão, freqüência) e história detalhada de tratamento psiquiátrico prévio. Todos os terapeutas receberam 30 horas de treinamento teórico-prático sobre o protocolo, e o preenchimento foi baseado nas entrevistas de avaliação (em média quatro), sendo realizado pelo terapeuta com auxílio de seu supervisor. As patologias em EIXO I foram avaliadas por entrevista clínica supervisionada. 
Tabela 1 - Critérios de indicação

\begin{tabular}{ll}
\hline 1 & Busca espontânea \\
2 & Disponibilidade de tempo \\
3 & Atividade profissional \\
4 & Inteligência \\
5 & Boa adaptação prévia \\
6 & Teste de realidade \\
7 & Pessoa significativa \\
8 & Qualidade das relações de objeto \\
9 & Expectativas realistas \\
10 & Sofrimento psíquico \\
11 & Curiosidade psíquica \\
12 & Pensa psicologicamente \\
13 & Tolerância à frustração \\
14 & Difusão de identidade \\
15 & Conflitos com dependência \\
16 & Descontrole de impulsos \\
17 & Assume responsabilidades \\
18 & Fator desencadeante \\
19 & Conflito focal identificável \\
20 & Motivação para mudança \\
21 & Aliança terapêutica \\
\hline
\end{tabular}

Os pacientes foram encaminhados por outros programas do Ambulatório do Serviço de Psiquiatria (por haver sintomatologia ou sofrimento residual após otimização do tratamento medicamentoso) ou diretamente do Ambulatório de Triagem (que recebe os pacientes da rede primária). Pode-se dizer que, havendo indicação segundo critérios psicológicos, muitos pacientes com diagnóstico em Eixo I que já estavam em tratamento medicamentoso iniciaram psicoterapia (caracterizando tratamento combinado) ou foram encaminhados diretamente à psicoterapia quando a natureza da patologia implicava evidências de igual efetividade em relação ao tratamento medicamentoso (como, por exemplo, depressão leve a moderada).

Uma vez indicada a psicoterapia, seu planejamento foi baseado na hipótese psicodinâmica preliminar: conflito atual, funcionamento do paciente, conflito primário e foco de trabalho escolhido. Manuais de tratamento não foram utilizados para "prescrever" o roteiro do processo terapêutico. No entanto, um rigoroso treinamento teórico, acompanhado de supervisão sistemática, foi utilizado para o treinamento técnico. O objetivo da nãomanualização da psicoterapia foi o de manter um “setting naturalístico", implicando em menor rigidez e em um tratamento mais próximo do "mundo real”22,29.
Pelo mesmo motivo, não se excluiu pacientes com comorbidades. A freqüência da psicoterapia foi de uma a duas vezes por semana, dependendo da indicação e da disponibilidade de horários. Os terapeutas foram supervisionados por psicoterapeutas experientes (15 a 40 anos de experiência em psicoterapia de orientação analítica), por meio de entrevistas dialogadas.

\section{Instrumentos padronizados}

\section{Self Report Questionnaire (SRQ)}

O SRQ é um instrumento auto-aplicável, validado para o português brasileiro, que avalia a gravidade clínica (sintomas) através de 20 questões do tipo "sim/ não” e é comumente usado para rastreamento da presença de transtorno psiquiátrico e avaliação de gravidade em nosso meio ${ }^{30,31}$. Para a classificação nas categorias "alta probabilidade de doença psiquiátrica" e "baixa probabilidade de doença psiquiátrica", foram utilizados os pontos de corte definidos no estudo de validação da versão brasileira do SRQ ${ }^{32}$.

\section{Diagnóstico segundo DSM-IV-TR}

O diagnóstico foi realizado ao longo das entrevistas de avaliação. Os terapeutas que realizaram as entrevistas tinham, pelo menos, 1 ano de treinamento sistemático na realização de diagnósticos segundo o DSM-IV-TR, com supervisão em internação psiquiátrica e atendimento ambulatorial, além de experiência com instrumentos estruturados de diagnóstico. Optou-se pela entrevista (considerada, por definição, padrão-ouro) no lugar de instrumentos padronizados, devido à experiência dos avaliadores e ao número significativo de entrevistas de avaliação, aumentando o tempo de contato com o paciente para a realização adequada do diagnóstico ${ }^{28}$.

\section{Global Assessment of Functioning (GAF)}

Corresponde ao Eixo V do DSM-IV-TR. É uma escala em que o clínico avalia o funcionamento do paciente quanto à presença de sintomas, funcionamento social, ocupacional ou escolar, relações interpessoais, comportamento, capacidade de autocuidado, risco de agressão ou suicídio, pensamento e capacidade de julgamento, entre outros. O escore resultante varia de 1 a 100, correspondendo zero a informações inadequadas para o preenchimento da escala ${ }^{28}$.

\section{Defensive Style Questionnaire (DSQ-40)}

O DSQ-40 é uma escala auto-aplicável, validada para o português brasileiro, com 40 questões que verificam o grau de utilização de 20 mecanismos de defesa, gerando escores para o fator maduro, imaturo 
e neurótico, que refletem o grau de utilização dos mecanismos pertencentes a cada categoria ${ }^{33}$.

\section{Escala de Funcionamento Defensivo (EFD/DSM- IV-TR)}

Escala em que o terapeuta deve escolher, pontuando de forma hierárquica, os sete mecanismos de defesa usados com maior freqüência dentre os 25 listados. O funcionamento defensivo do paciente é classificado, posteriormente, em um dos seguintes níveis: alto nível adaptativo, nível de inibições mentais (formação de compromisso), nível de leve distorção da imagem, nível da negação, nível de importante distorção da imagem, nível da ação e nível da desregulação defensiva. Um glossário padronizado é utilizado para homogeneizar a conceitualização de cada um dos mecanismos de defesa, bem como dos diferentes níveis defensivos ${ }^{34}$. Neste estudo, o nível defensivo foi determinado por consenso entre dois avaliadores, utilizando-se, além da pontuação das defesas, as descrições referentes ao funcionamento psíquico que faziam parte do protocolo de avaliação.

Posteriormente, os sete níveis foram agrupados em três categorias, considerando-se o grau de adaptação gerado pelo uso predominante do conjunto de defesas: a) categoria I - níveis 1 e 2 do DSM-IV-TR; b) categoria II - nível 3; e c) categoria III (menor adaptação) - níveis 4, 5, 6 e 7. Pode-se considerar que o grau de egodistonia, ou seja, quanto do seu sofrimento o paciente atribui ao seu estilo defensivo ou "jeito de ser", tende a ser menor na categoria III, pois esta engloba grupos de defesa fortemente ligados à negação, projeção, cisão, atuação, etc.

\section{World Health Organization Quality of Life Bref (WHOQOL-Bref)}

A percepção do paciente de sua qualidade de vida foi avaliada através da WHOQOL-Bref, escala desenvolvida em 1998 pelo Grupo de Qualidade de Vida da Organização Mundial de Saúde e validada para o português em 2000 por Fleck et al. Consiste de um instrumento auto-aplicável, composto por 26 questões, abreviado da WHOQOL-100. As duas primeiras são sobre qualidade de vida geral e satisfação com a saúde. Neste estudo, optou-se por avaliar cada uma das duas questões em separado, por tratarem de aspectos diferentes em relação à forma que o paciente vê a si mesmo e ao mundo. As 24 questões restantes representam os quatro domínios do instrumento original: físico, psicológico, relações sociais e meio ambiente ${ }^{35}$.

O protocolo e a EFD foram preenchidos pelo terapeuta, com auxílio de seu supervisor, ao longo do processo de avaliação da indicação da psicoterapia.
Uma vez realizada a indicação, cada paciente foi contatado por um auxiliar de pesquisa (estudante de medicina ou psicologia), e a aplicação do SRQ, DSQ40 e WHOQOL-Bref (auto-aplicáveis) foi realizada no HCPA, conforme horário conveniente para o paciente.

\section{Análise estatística e aspectos éticos}

Os grupos foram comparados através do teste $t$ de Student e o teste exato de Fisher, enquanto as correlações foram investigadas através dos testes de Pearson e Spearman. Foi realizada regressão linear para controlar a presença de diagnóstico em Eixo III em relação à satisfação com a saúde. O trabalho foi aprovado pelo Comitê de Ética e Pesquisa do HCPA (protocolo $n^{\circ}$ GPPG-HCPA 05-160), e todos os pacientes e seus terapeutas preencheram consentimento informado. A análise dos dados foi realizada utilizando-se o pacote estatístico SPSS 13.0, com o auxílio do Grupo de Pesquisa e Pós-Graduação do HCPA.

\section{Resultados}

Foram incluídos no estudo 47 pacientes do sexo feminino e nove do sexo masculino. A média de idade foi de $37,5 \pm 11,9$ anos; a renda média, 3,3 $\pm 2,8$ salários mínimos nacionais; e a escolaridade, $11,8 \pm 3,1$ anos de estudo completos. Trinta e dois pacientes foram encaminhados de outros ambulatórios, e 24, diretamente da triagem.

Dos 56 pacientes incluídos, um homem $(11,1 \%$ dos homens) e seis mulheres (12,8\% das mulheres) abandonaram o tratamento antes de 3 meses, totalizando $12,5 \%$ da amostra. Não houve diferença entre esse grupo $(n=7)$ e o que permaneceu em tratamento por mais de 3 meses $(\mathrm{n}=49)$ quanto à idade $(\mathrm{p}=0,32)$, ao estado civil $(\mathrm{p}=0,90)$, à presença de filhos $(\mathrm{p}=0,51)$ e a ser ou não praticante de alguma religião $(p=0,14)$. Quanto aos escores basais de gravidade, não houve diferença em relação ao SRQ $(10,71 \pm 3,5$ versus $11,47 \pm 4,8 ; p=0,69), G A F^{36}$ $(59,19 \pm 16,1$ versus $59,17 \pm 22,0 ; \mathrm{p}=0,99)$ e domínios da WHOQOL-Bref (físico: $\mathrm{p}=$ 0,56; psicológico: $\mathrm{p}=$ 0,73 ; relações sociais: $p=0,69$; e ambiente: $p=0,84$ ). Não houve diferença quanto à percepção da qualidade de vida geral $(p=0,96)$. Embora os parâmetros de gravidade tenham sido semelhantes, os pacientes que abandonaram a psicoterapia estavam mais satisfeitos com sua saúde $(\mathrm{p}<0,01)$ no início do tratamento, mesmo quando controlado para presença de diagnóstico em Eixo III $(\mathrm{p}<0,0001)$.

Houve uma tendência a menor renda pessoal (3,6 $\pm 2,9$ versus $1,50 \pm 1,4$ salários mínimos nacionais; $\mathrm{p}$ 
$=0,09)$ e menor escolaridade $(12,0 \pm 3,0$ versus $9,7 \pm 2,8$ anos de estudo; $\mathrm{p}=0,08$ ) no grupo do abandono. $\mathrm{O}$ grupo que permaneceu em tratamento apresentou maior uso de defesas maduras na avaliação inicial, segundo o DSQ-40 (4,5 $\pm 1,5$ versus $3,8 \pm 1,4)$, mas esse achado não foi significativo ( $\mathrm{p}=0,23)$.

Em relação aos critérios de indicação da psicoterapia, o grupo dos que permaneceram em tratamento teve maior proporção de indivíduos com boa adaptação prévia (81,6 versus $42,9 \%$; $\mathrm{p}=0,04)$ e inteligência clinicamente na média ou superior (96 versus $57,1 \% ; p=0,05)$. No único caso em que se avaliou a aliança terapêutica como ruim, o paciente abandonou o tratamento.

Um dos pacientes no grupo do abandono, quando perguntado sobre o motivo da interrupção, alegou que já havia atingido seus objetivos (apesar do curto tempo de tratamento) e que não sentia mais necessidade de seguir em psicoterapia. Quando as análises estatísticas foram repetidas, sem considerar esse caso, a menor escolaridade no grupo do abandono tornou-se significativa $(12,2 \pm 3,0$ versus $9 \pm 2,5 ; p=0,036)$

De acordo com a $\mathrm{EFD}^{34}$, maior proporção de pacientes que interromperam o tratamento foi classificada em níveis imaturos, particularmente no nível da negação (Tabela 2). Quando agrupados em três categorias - sendo I maior adaptação e III defesas mais desadaptativas e maior egossintonia -, 55\% dos pacientes que permaneceram em tratamento foram classificados na categoria III, enquanto $85,7 \%$ dos que abandonaram foram incluídos nessa categoria $(p=0,13)$. Enquanto pacientes da categoria III que permaneceram em tratamento tiveram uma percepção da sua qualidade de vida geral pior do que os demais pacientes $(37,0 \pm 25,9$ versus 51,1 $\pm 19,3$; $p=0,04$ ), isso não ocorreu com os pacientes incluídos nessa categoria que abandonaram o tratamento $(51,1 \pm 18,8)$.

Tabela 2 - Níveis defensivos conforme a Escala de Funcionamento Defensivo do Manual de Diagnóstico e Estatística das Perturbações Mentais, 4ª edição, texto revisado (EFD/DSM-IV-TR)

\begin{tabular}{|c|c|c|c|c|}
\hline & \multicolumn{2}{|c|}{$\begin{array}{l}\text { Não-abandono } \\
\quad(n=49)\end{array}$} & \multicolumn{2}{|c|}{$\begin{array}{l}\text { Abandono } \\
(\mathrm{n}=7)\end{array}$} \\
\hline & $\mathrm{n}$ & $\%$ & $\mathbf{n}$ & $\%$ \\
\hline \multicolumn{5}{|l|}{ Nível 1} \\
\hline Alto nível adaptativo & 0 & 0 & 0 & 0 \\
\hline \multicolumn{5}{|l|}{ Nível 2} \\
\hline Inibições mentais & 12 & 24,5 & $1 *$ & 14,3 \\
\hline \multicolumn{5}{|l|}{ Nível 3} \\
\hline Leve distorção da imagem & 10 & 20,4 & 0 & 0 \\
\hline \multicolumn{5}{|l|}{ Nível 4} \\
\hline Negação & 11 & 22,4 & 3 & 42,9 \\
\hline \multicolumn{5}{|l|}{ Nível 5} \\
\hline Importante distorção da imagem & 7 & 14,3 & 1 & 14,3 \\
\hline \multicolumn{5}{|l|}{ Nível 6} \\
\hline Ação & 9 & 18,4 & 2 & 28,6 \\
\hline \multicolumn{5}{|l|}{ Nível 7} \\
\hline Desregulação defensiva & 0 & 0 & 0 & 0 \\
\hline
\end{tabular}

Utilizando o ponto de corte do SRQ para deteçãa de possível doença psiquiátrica na população brasileira, 85,7\% dos pacientes que não permaneceram em psicoterapia tinham alta probabilidade de doença. Segundo a classificação do DSM-IV-TR ${ }^{36}$, todos tinham pelo menos um diagnóstico em Eixo I ou Eixo II (Tabela 3). 
Tabela 3 - Diagnóstico conforme o Manual de Diagnóstico e Estatística das Perturbações Mentais, $4^{\mathrm{a}}$ edição, texto revisado (DSM-IV-TR)

\begin{tabular}{|c|c|c|c|c|}
\hline & \multicolumn{2}{|c|}{ Não-abandono } & \multicolumn{2}{|c|}{ Abandono } \\
\hline & Freqüência & $\%$ & Freqüência & $\%$ \\
\hline \multicolumn{5}{|l|}{ Eixo I } \\
\hline Nenhum diagnóstico & 12 & 24,5 & 1 & 14,3 \\
\hline Pelo menos um diagnóstico & 37 & 75,5 & 6 & 85,7 \\
\hline Depressão maior & 14 & 28,6 & 1 & 14,3 \\
\hline Transtorno bipolar & 6 & 12,3 & 2 & 28,6 \\
\hline Estresse pós-traumático & 4 & 8,2 & 0 & 0 \\
\hline Dependência química & 3 & 6,1 & 0 & 0 \\
\hline Ansiedade generalizada & 3 & 6,1 & 0 & 0 \\
\hline Transtorno do pânico & 3 & 6,1 & 0 & 0 \\
\hline Transtorno alimentar & 2 & 4,1 & 0 & 0 \\
\hline Fobia social & 1 & 2,0 & 0 & 0 \\
\hline Déficit de atenção e hiperatividade & 1 & 2,0 & 0 & 0 \\
\hline Transtorno de estresse agudo & 0 & 0 & 1 & 14,3 \\
\hline Transtorno somatoforme & 0 & 0 & 1 & 14,3 \\
\hline Transtorno sexual & 0 & 0 & 1 & 14,3 \\
\hline \multicolumn{5}{|l|}{ Eixo II } \\
\hline Sem transtorno de personalidade & 34 & 69,4 & 5 & 71,4 \\
\hline Com transtorno de personalidade & 15 & 30,6 & 2 & 28,6 \\
\hline Personalidade borderline & 9 & 18,4 & 1 & 14,3 \\
\hline Personalidade dependente & 4 & 8,2 & 1 & 14,3 \\
\hline Personalidade histriônica & 1 & 2,0 & 0 & 0 \\
\hline Personalidade obssessivo-compulsiva & 1 & 2,0 & 0 & 0 \\
\hline \multicolumn{5}{|l|}{ Eixo III } \\
\hline Sim & 22 & 44,9 & 4 & 57,1 \\
\hline Não & 27 & 55,1 & 3 & 42,9 \\
\hline \multicolumn{5}{|l|}{ Eixo IV } \\
\hline Sim & 41 & 83,7 & 5 & 71,4 \\
\hline Não & 8 & 26,3 & 2 & 28,6 \\
\hline Eixo V & $59,17 \pm 22,0$ & - & $59,19 \pm 16,1$ & - \\
\hline
\end{tabular}

Oito terapeutas eram homens, e 10, mulheres, sendo paciente e terapeuta do mesmo sexo em $58,9 \%$ das duplas. Não houve diferença quanto às taxas de abandono em relação àquelas em que o gênero do terapeuta e do paciente foram diferentes $(\mathrm{p}=0,99)$. Todos os casos de abandono foram levados à supervisão. Dos 24 pacientes encaminhados diretamente da triagem, 25\% abandonaram o tratamento, enquanto que apenas um paciente $(3,1 \%)$ dos que foram encaminhados de outro ambulatório não permaneceu em psicoterapia $(p=0,035)$. Nenhum paciente que interrompeu o tratamento tinha realizado psicoterapia de orientação psicanalítica no passado, enquanto que boa parte dos que permaneceram (25 pacientes) já a haviam realizado em algum momento da vida $(p=0,01)$.
Da mesma forma, seis dos sete pacientes do grupo do abandono nunca haviam realizado tratamento psiquiátrico, exceto a atual psicoterapia de orientação psicanalítica $(\mathrm{p}=0,02)$.

\section{Discussão}

A taxa de abandono (12,5\%) encontrada neste estudo é pequena quando comparada à literatura ${ }^{37}$. Isso pode refletir um processo mais criterioso da seleção para psicoterapia, que incluiu 21 critérios retirados da literatura e discutidos em painel de experts (Tabela 1). Além disso, houve também avaliação clínica e psicodinâmica cuidadosa para o planejamento da 
psicoterapia. Esse dado implica em uma das limitações deste estudo: o número pequeno de pacientes no grupo do abandono, resultando em importante probabilidade de erro tipo beta. No entanto, alguns achados foram ao encontro de aspectos freqüentemente discutidos na literatura, justificando a apresentação dos resultados. Outro possível limitador é o fato de os terapeutas estarem em período de formação; por outro lado, esses terapeutas foram submetidos a rigoroso treinamento técnico e supervisão sistemática, favorecendo a validade interna.

A similaridade dos dois grupos quanto à gravidade clínica (SRQ e GAF) sugere descartar, nesta amostra, o comprometimento clínico (sintomático) como possível causador da interrupção. No entanto, aparentemente de forma paradoxal, os pacientes que abandonaram o tratamento estavam mais satisfeitos com sua saúde no início da terapia, mesmo quando se controlou a presença de doenças em Eixo III. Por outro lado, quando se considera a variável psicodinâmica "mecanismos de defesa” (DSQ-40 e EFD), o grupo do abandono demonstrou um funcionamento defensivo mais imaturo em diversos parâmetros, apontando uma maior dificuldade em tomar contato com a realidade e lidar com estressores externos e internos. Isso está de acordo com outros estudos, que associaram um menor nível de insight e maior uso de defesas narcisistas a um pior prognóstico. Pode-se pensar, portanto, que os pacientes que abandonaram a psicoterapia, na nossa amostra, não se reconheciam como doentes. Mesmo que o objetivo da psicoterapia analítica seja justamente aumentar a capacidade de insight e melhorar o teste de realidade, dependendo da inexistência de maiores recursos de ego, níveis muito elevados de projeção e negação podem prejudicar a utilização do método, impedindo um melhor aproveitamento das interpretações e de outras intervenções.

A conscientização da responsabilidade do sujeito sobre sua situação atual é justamente o que torna possível modificá-la. A falta de motivação e a incapacidade persistente de reconhecer esses aspectos tornam o método psicodinâmico improdutivo, inviabilizando, justamente, o aumento da percepção sobre si mesmo, sobre os outros e sobre o mundo.

Uma tendência a maior escolaridade, melhor nível socioeconômico e inteligência avaliada clinicamente como média e superior no grupo que permaneceu em tratamento aponta para a importância de uma condição intelectual satisfatória para melhor aproveitamento da psicoterapia de orientação psicanalítica. Pode-se assumir que essas variáveis refletem a capacidade de abstração e simbolização necessárias ao processo. Corroborando a importância de um nível mínimo de organização do ego, encontramos, no grupo que permaneceu em tratamento, maior proporção de indivíduos com boa adaptação prévia, inteligência clinicamente na média ou superior, além de uma tendência a um menor número de pacientes com difusão de identidade. No único caso em que o terapeuta avaliou a aliança terapêutica como ruim, o paciente abandonou o tratamento, o que concorda com achados da literatura de que a aliança de trabalho é indispensável para o bom andamento da psicoterapia.

No presente estudo, não se observaram abandonos por causa de trocas de terapeuta, já que nenhum dos pacientes havia realizado psicoterapia anteriormente. Considera-se importante ressaltar o fato, pois a literatura o apresenta como um dos principais motivos de interrupção do tratamento em instituições de ensino. Como nenhum paciente que interrompeu o tratamento havia realizado tratamento psicoterápico anterior (contra mais de $50 \%$ dos que permaneceram), poder-se-ia pensar que esse grupo desconhecia os objetivos reais de um tratamento de orientação analítica. Além disso, como os pacientes que vieram diretamente da triagem abandonaram mais o tratamento, deve-se considerar a possibilidade de que a indicação da psicoterapia tenha sido melhor trabalhada pelos psiquiatras dos pacientes que foram encaminhados de outros programas. Por outro lado, esses mesmos pacientes haviam procurado menos tratamentos em geral, apesar de comprometimento clínico semelhante, reforçando a hipótese de que talvez se percebessem como menos doentes, menos necessitados ou, devido às características narcisistas presentes, com maior dificuldade de buscar ajuda. De acordo com outros estudos, pacientes com maior nível de insight buscam mais tratamentos e se beneficiam mais dos mesmos ${ }^{20,21}$.

Considerando que a aliança terapêutica tem sido a variável mais freqüentemente associada aos desfechos em psicoterapia, pode-se pensar os achados do estudo como sendo fatores potencialmente envolvidos na capacidade de se estabelecer uma aliança de melhor qualidade para a realização de um tratamento desse tipo $^{24}$. É importante ressaltar que, nesse caso, trata-se de uma aliança para a realização de trabalho psicodinâmico, em oposição a um tratamento que ofereça resultados rápidos e "mágicos". Maior capacidade de abstração e simbolização, menor uso de defesas imaturas e menos características narcísicas possibilitam buscar e receber ajuda de forma mais adequada e aumentam a chance de que o paciente tolere melhor a fase inicial do tratamento. No mesmo sentido, maior senso crítico, tanto sobre a própria condição de doente quanto sobre prejuízos secundários ao seu funcionamento em geral, colabora para a construção da aliança de trabalho analítico. Ao contrário, quando predominam defesas imaturas (principalmente negação e projeção), o aproveitamento da psicoterapia fica mais comprometido. 
Esses resultados sugerem que, independentemente do diagnóstico psiquiátrico do paciente, melhor definição de seus objetivos, do que ele espera do tratamento, de seu nível de motivação e de suas possibilidades de pensar psicologicamente são fatores essenciais que devem ser investigados criteriosamente na fase de avaliação, quando se considera qual a melhor modalidade de tratamento a ser indicada para aquele paciente em particular.

\section{Referências}

1. Leichsenring F. Are psychodynamic and psychoanalytic therapies effective?: A review of empirical data. Int J Psychoanal. 2005;86(Pt 3):841-68.

2. Durham R, Murphy T, Allan T, Richard K, Treliving L, Fenton G. Cognitive therapy, analytic psychotherapy and anxiety management training for generalised anxiety disorder. Br J Psychiatry. 1994;165(3):315-23.

3. Gallagher-Thompson D, Steffen A. Comparative effects of cognitivebehavioral and brief psychodynamic psychotherapies for depressed family caregivers. J Consult Clin Psychol. 1994;62(3):543-9.

4. Hardy G, Barkham M, Shapiro D, Stiles W, Rees A, Reynolds S. Impact of cluster $\mathrm{C}$ personality disorders on outcomes of contrasting brief psychotherapies for depression. J Consult Clin Psychol. 1995;63(6):997-1004.

5. Joyce A, Piper W. Interpretative work in short-term individual psychotherapy: an analysis using hierarchical linear modeling. J Consult Clin Psychol. 1996;64(3):505-12.

6. Piper W, Joyce A, McCallum M, Azim H. Interpretative and supportive forms of psychotherapy and patient personality variables. J Consult Clin Psychol. 1998;66(3):558-67.

7. Saunders D. Feminist-cognitive-behavioral and processpsychodynamic treatments for men who batter: interaction of abuser traits and treatment models. Violence Vict. 1996;11(4):393-414.

8. Leichsenring F, Rabung S, Leibing E. The efficacy of short-term psychodynamic psychotherapy in specific psychiatric disorders: a meta-analysis. Arch Gen Psychiatr. 2004;61(12):1208-16.

9. Valbak K. Suitability for psychoanalytic psychotherapy: a review. Acta Psychiatr Scand. 2004;109(3):164-78.

10. Keidann CE, Dal Zot JS. Avaliação. In: Eizerik CL, Schestatski SS, Aguiar RW, eds. Psicoterapia de orientação analítica: fundamentos teóricos e clínicos. 2 ed. Porto Alegre: Artmed; 2005. p. 193-205.

11. Maat SD, Dekker J, Schoevers R, Jonghe FD. The effectiveness of long-term psychotherapy: methodological research issues. Psychother Res. 2007;17(1):59-65.

12. Lambert M. Presidencial address: what we have learned from a decade of research aimed at improving psychotherapy outcome in routine care. Psychother Res. 2007;17(1):1-14.

13. Zimmerman DE. Planejamento em psicoterapia dinâmica. Rev Psiquiatr RS. 1982;4(3):252-63.

14. Fonagy P. Psychoanalysis today. World Psychiatry. 2003;2(2):7380 .

15. Kandel ER. A biologia e o futuro da psicanálise. Rev Psiquiatr RS. 2003;25(2):139-65.

16. Freud S. Edição eletrônica da standard brasileira das obras psicológicas completas de Sigmund Freud. Versão original de 196980. Rio de Janeiro: Imago; 1980.

17. Urtiaga ME, Almeida GD, Vianna MED, Santos MVDS, Botelho S. Fatores preditivos de abandono em psicoterapias: um estudo na clínica Sérgio Abuchaim. J Bras Psiquiatr. 1997;46(5):279-83.
18. Lhullier AC, Nunes MLT, Antochevis AF, Porto AM, Figueiredo D. Mudança de terapeuta e abandono da psicoterapia em uma clínicaescola. Rev Aletheia. 2000;11:7-11.

19. Stone MH. Treatment of borderline patients: a pragmatic approach. Psychiatr Clin North Am. 1990;13(2):265-85.

20. Junkert-Tress B, Tress W, Hildenbrand G, et al. [Premature termination-a multifactoral phenomenon]. Psychotherapie, Psychosomatik, medizinische Psychologie. 2000;50(9-10):35165.

21. Hoglend P, Engelstad V, Sorbye O, Heyerdahl O, Amlo S. The role of insight in exploratory psychodynamic psychotherapy. Br J Med Psychol. 1994;67( Pt 4):305-17.

22. Garfield SL, Bergin AE. Handbook of psychotherapy and behavior change. 5th ed. New York: John Wiley \& Sons; 2005.

23. Marcolino JAM, Iacoponi E. O impacto inicial da aliança terapêutica em psicoterapia psicodinâmica breve. Rev Bras Psiquiatr. 2003;25(2).

24. Barber JP, Connolly MB, Crits-Christoph P, Gladis L, Siqueland L. Alliance predicts patients outcome beyond in-treatment change in symptoms. J Consult Clin Psychol. 2000;68(6):1027-32.

25. Martin DJ, Garske JP, Davis MK. Relation of the therapeutic alliance with outcome and other variables: a meta-analytic. J Consult Clin Psychol. 2000;68(3):438-50.

26. Schestatsky S. Introdução ao planejamento em psicoterapia. In: Eizirik C, Aguiar R, Schesatsky S. Psicoterapia de orientação analítica: teoria e prática. Porto Alegre: Artmed; 1989:71-8.

27. Hauck S, Gomes FG, Cervieri A, et al. Desenvolvimento do instrumento para avaliação da indicação de psicoterapia psicanalítica: modelagem de equação estrutural. Rev Bras Psicoter. 2006;8(2/3):155-70.

28. American Psychiatric Associaton. Avaliação multiaxial. In: Manual diagnóstico e estatístico de transtornos mentais (DSM-IV-TR). 4 ed. Porto Alegre: Artmed; 2002. p. 59-69.

29. Eizerik C, Schestatski SS, Aguiar RW, eds. Psicoterapia de orientação analítica: fundamentos teóricos e clínicos. 2 ed. Porto Alegre: Artmed; 2005.

30. Almeida O, Forlenza O, Lima N, et al. Psychiatric morbidity among the elderly in a primary care setting - report from a survey in São Paulo, Brazil. Int J Geriatr Psychiatry. 1997;12(7):728-36.

31. Mari JJ, Williams P. A comparison of the validity of two psychiatric screening questionnaires (GHQ-12 and SRQ-20) in Brazil, using Relative Operating Characteristic (ROC) analysis. Psychol Med. 1985;15(3):651-9.

32. Mari JJ, Williams P. A validity study of a Psychiatric Screening Questionnaire (SRQ-20) in Primary Care in the city of São Paulo. Br J Psychiatry. 1986;148:23-6.

33. Blaya C, Kipper L, Heldt E, et al. [Brazilian-Portuguese version of the Defense Style Questionnaire (DSQ-40) for defense mechanisms measure: a preliminary study]. Rev Bras Psiquiatr. 2004;26(4):2558 .

34. American Psychiatric Associaton. Eixo proposto para estudos adicionais: Escala de Funcionamento Defensivo In: American Psychiatric Associaton. DSM-IV-TR ${ }^{\mathrm{TM}}$ - Manual diagnóstico e estatístico de transtornos mentais. Porto Alegre: Artmed; 2002. p. 754-9.

35. Fleck MP, Louzada S, Xavier M, et al. [Application of the Portuguese version of the abbreviated instrument of quality life WHOQOLbref]. Rev Saude Publica. 2000;34(2):178-83.

36. American Psychiatric Associaton. DSM-IV-TR ${ }^{\mathrm{TM}}$ - Manual diagnóstico e estatístico de transtornos mentais. 4 ed. Porto Alegre: Artmed; 2002.

37. Jung SI, Fillippon APM, Nunes MLT, Eizirik CL. História recente e perspectivas atuais da pesquisa de resultados em psicoterapia psicanalítica de longa duração. Rev Psiquiatr RS. 2006;28(3):298312. 